\title{
Acoso escolar
}

\author{
P. Armero Pedreira ${ }^{a}$, B. Bernardino Cuesta ${ }^{b}$, C. Bonet de Luna ${ }^{c}$ \\ aMIR-Pediatría. Hospital Universitario La Paz. Madrid. España. \\ ${ }^{b}$ MIR-Pediatría. Hospital Universitario Niño Jesús. Madrid. España. \\ cPediatra. CS Segre. Madrid. España.
}

\section{Resumen}

El bullying, acoso escolar o violencia entre iguales, produce mucho sufrimiento en quienes lo padecen. Aunque tradicionalmente se suele enfatizar la relación acosador-acosado, hay más actores. Tanto por la angustia que causa a la víctima, por el refuerzo que recibe el acosador cuyas conductas agresivas quedan impunes, como por la insensibilización o indefensión aprendida de los espectadores, debe ser abordado como un problema grupal y social. El mejor tratamiento es la prevención y se debe trabajar desde el colegio, incluyendo a los alumnos, las familias y en algunos casos al pediatra (en cuanto promotor de la salud física y mental en la infancia).

Palabras clave: Acoso escolar. Estrés. Ansiedad.

\section{Bullying}

Abstract

Bullying or peer violence causes much suffering. Although traditionally the relationship between bully-bullied is the most emphasized in literature, there are more players. Because of the distress caused to the victim, the reinforcement received by the stalker whose aggressive behavior goes unpunished, and the learned helplessness of spectators, it must be tackled as a group and social problem. Prevention is the best cure and we should work as a team in schools, including students, families and in some cases the pediatrician (as a promoter of physical and mental health in childhood).

Key words: Bullying. Stress. Anxiety.

\section{Introducción}

El maltrato entre iguales no es algo nuevo, pero ha empezado a ser una preocupación social por la importancia mediática que ha suscitado el suicidio de algunos afectados. Se presenta principalmente en la escuela primaria y aunque va disminuyendo a medida que los sujetos maduran, sigue habiendo acoso tanto en el medio laboral como en el familiar (entre adultos). Y es que la violencia forma parte de la historia de la humanidad.

A diferencia de los animales, que presentan conductas agresivas para defen-

Paula Armero Pedreira, elanor27@hotmail.com

Las autoras declaran no presentar conflictos de intereses en relación con la preparación y publicación de este artículo. 
der territorio, comida o el acceso a las hembras, los humanos podemos ser violentos sin necesidad, con intención de dañar a otro, por diversión, por estupidez o por un mal manejo de poder o del conflicto ${ }^{1,2}$.

Aunque dirimir si la condición humana es violenta genéticamente o por aprendizaje excede los objetivos de este artículo, releer la novela de W. Golding, El Señor de las moscas, está al alcance de todos.

\section{Definición}

Inicialmente descrito por Heineman en 1969 y Olweus en 1973, es una forma de violencia que se da entre compañeros y por tanto frecuente en contexto escolar. Existen distintos términos para referirnos a este fenómeno: acoso escolar, bullying (del inglés bully, que significa "matón") o violencia entre iguales. Este último parece el más adecuado. La definición de Olweus ${ }^{3}$ es muy utilizada: “Un estudiante es acosado o victimizado cuando está expuesto de manera repetitiva a acciones negativas por parte de uno o más estudiantes, sin capacidad para defenderse".

Para definirlo de forma más precisa, es necesario que los sujetos posean intencionalidad (puede no estar presente antes de los siete años), de forma que las acciones sean deliberadamente hostiles.
Tiene que repetirse durante un periodo de tiempo (no ser algo puntual) y causar daño a la victima que se encuentra sola (en situación de desamparo) e incapaz de resolverlo. No hay (generalmente) provocación por parte del alumno que lo sufre. Es decir, no toda confrontación, pelea o insulto es acoso escolar.

La forma en que se comete puede ser verbal, física o de aislamiento social hacia la víctima (tabla 1). Los espectadores (otros alumnos) son clave, ya que es a ellos a quien el acosador quiere mostrar su poder. Con su silencio permiten la perpetuación de estas acciones (apoyando o no al agresor). En cuanto a los contenidos, pueden ser racistas si hacen referencia al origen étnico del sujeto, sexuales si incluyen burlas con connotaciones sexuales, u homófobos si tienen que ver con la supuesta orientación sexual. Si el medio utilizado son los mensajes a través del teléfono móvil o el ordenador, se habla de "bullying digital". Este puede llegar a ser devastador, ya que la víctima no tiene un lugar donde estar en paz.

\section{Magnitud y gravedad del problema}

Solo existe un estudio oficial a nivel nacional que estime la incidencia y prevalencia del acoso escolar, el realizado por el Defensor del Pueblo ${ }^{4}$, si bien el estudio más amplio en cuanto a volumen de po- 
Tabla 1. Tipo de agresiones hacia la víctima

Agresiones físicas:

- Directas: pegar, dar empujones, amenazar, intimidar...

- Indirectas: esconder, romper, robar objetos de la víctima

Agresiones verbales:

- Directas: vocear, burlarse, insultar y poner motes

- Indirectas: hablar mal a sus espaldas, hacer que lo oiga "por casualidad", enviarle notas groseras, cartas, hacer pintadas, difundir falsos rumores, etc.

Agresiones relacionales (exclusión social):

- Directas: exclusión deliberada de actividades, impedir su participación (se ve a la víctima alejada en el patio, en clase es evitada, siempre queda sin pareja)

- Indirectas: ignorarla, hacer como si no estuviera o como si fuera transparente

blación encuestada es el realizado por Oñate y Piñuel en 2007 (Informe Cisneros X) $)^{5}$ que incluyó a 24990 niños de 14 comunidades autónomas españolas, entre $2 .^{\circ}$ de Primaria (siete años) y $1 .^{\circ}$ de Bachillerato (17 años).

El estudio del acoso en España se inicia en nuestro país por Vieira, Fernández y Quevedo en $1989^{6}$ en Madrid, donde 1200 alumnos de ocho, diez y 12 años de diez centros rellenaron un cuestionario de elección múltiple, diseñado por los autores. Como resultados destacan que el $17,3 \%$ de los alumnos intimidaba a sus compañeros, mientras que el $17,2 \%$ había sido intimidado en el último trimestre. Las formas de acoso más frecuentes eran las agresiones verbales $(19,3 \%)$, el robo $(13,9 \%)$ y las intimidaciones físicas $(12,7 \%)$.

Ortega ${ }^{7,8}$ realizó para la Junta de Andalucía diversos informes (entre 1992 y
1996) sobre la presencia de malos tratos y violencia entre iguales en las escuelas, utilizando como base el Cuestionario Olweus. Con los datos obtenidos, este cuestionario evalúa a los alumnos involucrados en problemas de violencia, considerando su propia adscripción dentro de las categorías de víctima, intimidador, intimidador-victimizado o espectador. Esto se define a través de una serie de aseveraciones sobre acciones (tanto activas como pasivas), consideradas parte del acoso escolar, que son explicadas previamente a los alumnos.

El Informe Cisneros X del 2007, (reseñado anteriormente), concluye que la tasa global de acoso (intenso y muy intenso) es del 23,3\%. Se basan en la realización del Test AVE (Acoso y Violencia Escolar), donde se incluye tanto violencia física (agresiones, intimidación y amenazas) como psicológica (hostigamiento, 
manipulación, bloqueo, exclusión social y coacción) e incorpora escalas clínicas que evalúan el daño psicológico consecuente (por ejemplo, en forma de somatizaciones, flashback, disminución de la autoestima, etc.).

Estos autores señalan que en las víctimas que han sufrido acoso escolar frecuente y no han sido adecuadamente atendidas, hay un riesgo cuatro veces mayor de estrés postraumático y ansiedad grave. La ideación suicida recurrente, es cinco veces mayor. Las conductas de violencia psicológica basadas en la burla y la exclusión social son las que más incrementan este riesgo. El tipo de agresión más frecuente suele ser verbal, pero el aislamiento social tiene una frecuencia similar, especialmente a edades más jóvenes. Los diferentes estudios evidencian un descenso paulatino de la violencia a medida que aumenta la edad (y el curso).

A la hora de analizar estos estudios es importante valorar adecuadamente las definiciones de acoso escolar que emplean y la forma en que se han obtenido los datos. Esto facilita que los estudios puedan ser comparables. Por ejemplo, parece importante analizar, si los cuestionarios permiten distinguir de forma válida y fiable a las víctimas (no todos los afectados se consideran a sí mismos como tales), acosadores (si se ha analiza- do la intencionalidad o solo se ha basado en la percepción por parte de la víctima) y espectadores; si en el estudio se utilizaron cuestionarios adaptados según las edades (ya que los comportamientos cambian), etc. La propia definición de Olweus solo se refiere a acciones negativas, sin reflejar la repercusión que esos actos conllevan, ni define el tiempo necesario para que esas acciones se consideren acoso. Es decir, Olweus define el acoso en función de que un comportamiento se produzca, independientemente de que dicho comportamiento provoque (o no) un efecto en el otro. Pero el acoso no es solo una conducta intencionada y violenta, sino que las acciones tienen que tener una clara repercusión; es un tipo de relación, en el que la conducta de uno (o más) tiene consecuencias indeseables en el otro. Por ello, el tratamiento no es solo de las personas, sino de la relación que estas desarrollan entre sí.

Para valorar la magnitud de este problema ayudaría establecer unas definiciones comunes, con cuestionarios protocolizados y actuaciones consensuadas y globales a nivel nacional.

\section{Sintomatología}

La víctima puede presentar un rango amplio de problemas generalmente en- 
cuadrables en los trastornos de ansiedad: fobia escolar o social, crisis de angustia, trastornos de apetito y de sueño (pudiendo llegar al trastorno de estrés postraumático). También hay sensación de indefensión, baja autoestima, retraimiento social y cuadros depresivos (llegando algunos a ideación suicida o a actuarlo). Es habitual que presenten somatizaciones y que el rendimiento escolar decaiga. Como pediatras, profesores y padres hay que sospecharlo: a veces los niños lo cuentan, pero no siempre. Pueden tener miedo por las represalias que prevén de sus compañeros. Otros piensan que son cobardes porque no saben defenderse, tienen vergüenza y lo esconden a los propios padres. Algunos no lo dicen porque no creen que lo que les está pasando sea un maltrato. Creen que este consiste en ataques físicos, pero no insultos ni conductas de exclusión o aislamiento (que pueden tener efectos mucho más graves). Algunos creerán que se lo merecen, ya que está tan aceptado por sus iguales que les parece inevitable. Finalmente otros acaban resignándose tras denunciarlo, ya que los adultos responsables le restan importancia y son etiquetados de "blandos", "acusicas" o "cobardes". En todos los casos el malestar se expresa. Por la ansiedad y el sufrimiento que padecen las víctimas es frecuente un bajo rendimiento escolar, además de comportamientos disfuncionales que actúan como feedback para perpetuar el acoso y facilitar estos errores de atribución. En la tabla 2 se resumen algunos comportamientos que deben hacernos sospechar que puede haber bullying.

\section{Los perfiles}

Algunos autores describen características comunes y rasgos de personalidad que se observan con frecuencia, pero desde nuestro punto de vista es algo controvertido hablar de perfiles de víctima, espectador o acosador, porque todo escolar puede sufrirlo, ser testigo o acosar.

Según diversos autores, la víctima no suele ser popular. Es habitualmente tímido, introvertido, con una personalidad débil o algún rasgo físico diferente hacia el cual pueden ir dirigidas las burlas. La falta de habilidad social, si además está presente, lo favorece. Para Olweus, los padres sobreprotectores generan niños dependientes y apegados a ellos, que son más vulnerables, pero en ocasiones esto puede ser el efecto del acoso?.

El acosador se puede encuadrar en el líder del grupo, no por su carisma sino por su fortaleza. No suele ser buen estudiante, es frecuentemente repetidor (más mayor y corpulento), puede cum- 
Tabla 2. Signos y sintomas sugestivos de maltrato

- Somatizaciones (por la mañana se encuentra mal, tiene dolor de cabeza, de tripa...; en la escuela dice que no se encuentra bien y pide que le vayan a recoger...)

- Cambios de hábitos (hace "pellas", no quiere ir a la escuela, quiere que le acompañen o cambia la ruta habitual, no quiere ir en el transporte escolar, no quiere salir con los amigos...)

- Cambios en la actitud hacia las tareas escolares (baja el rendimiento académico)

- Cambios de carácter (está irritable, se aísla, se muestra introvertido, más arisco, angustiado o deprimido, empieza a tartamudear, pierde confianza en sí mismo)

- Alteraciones en el apetito (pierde el apetito, o vuelve con hambre porque le han quitado el bocadillo o el dinero)

- Alteraciones en el sueño (grita por la noche, tiene pesadillas, le cuesta dormirse o se despierta muy pronto...)

- Vuelve a casa regularmente con la ropa o el material roto. Tiene moratones, heridas o cortes inexplicables

- Empieza a amenazar o a agredir a otros niños o hermanos menores

- Rehúsa decir por qué se siente mal e insiste en que no le pasa nada. Da excusas extrañas para justificar todo lo anterior

- En casos graves puede llegar a tener ideaciones o a realizar intentos de suicidio

plir criterios del trastorno por déficit de atención (TDA) con impulsividad por lo que no piensa en las consecuencias de sus actos. Es interesante este rasgo, ya que muchos niños que presentan criterios de TDA pueden oscilar entre acosar y ser acosados. Suelen actuar por "prontos" y resuelven mal los conflictos. Muchas veces descargan sus frustraciones con el débil del grupo de forma inapropiada, ya que carecen de empatía y sentimiento de culpabilidad. No se debe olvidar que muchas veces el acosador proviene de hogares disfuncionales. Olweus describe dos perfiles: el agresor activo, que es el que arremete directa y personalmente, y el indirecto, que es el verá reforzada. que desde la sombra dirige el comportamiento de sus seguidores.

Los espectadores pueden tener cualquier característica. Pueden ser alumnos modelo que de alguna manera acaban interiorizando errores de atribución hacia la víctima ("se lo merece") o tienen miedo de acabar ellos siendo las víctimas, perdiendo popularidad al defenderle. Olweus habla del modelo de contagio social, que actúa en los grupos y afecta a todos pero sobre todo a aquellos que no tienen espíritu crítico, son inseguros, dependientes y no cuentan para el resto de sus compañeros. Si además la conducta violenta no es censurada y tiene éxito, se 


\section{Qué se debe hacer}

Sospecharlo y preguntarlo en las revisiones habituales de salud. Si hay indicios, creer de entrada al niño (y a los padres), pero teniendo serenidad y sentido común. Hay una diferencia importante entre las peleas diarias puntuales de los chicos, las bromas pesadas entre compañeros y el auténtico maltrato. Por tanto, ante una sospecha fundada, hay que contener y apoyar psicológicamente al niño, preguntándole directamente y asegurando que se le va ayudar. Los padres se encuentran ante algo muy culpabilizante, doloroso y ansiógeno, ya que sienten que no han sabido proteger a su hijo. Como no saben cómo manejarlo (ya que escapa a su conocimiento y control) pueden tener reacciones de reproche hacia él, ya que "la tristeza y la furia a veces se intercambian la ropa". Otros pretenden "hacer justicia" siendo violentos con el agresor o con el colegio. Hay algunas guías para padres que pueden ser de utilidad $^{10}$.

Hay que informar al niño que no es su culpa y que ha hecho bien en denunciarlo. Hay que hacerle entender, que los adultos responsables estamos para ayudar e intentar resolver la situación. Una buena historia incluye saber desde cuándo ocurre, dónde y quién ha estado implicado, quién lo ha visto y a quién se lo ha contado. Hay que evaluar el grado de ansiedad del niño y la gravedad de la situación. Es importante que los padres (y el niño si prefiere), apoyados por el pediatra, se hagan cargo y hablen con el colegio (tutor o director de centro) ya que son los profesores quienes deben asegurar que esto no ocurra. Es un derecho de los alumnos estar seguros en los centros escolares y a la vez, es un deber de los centros velar por la seguridad de sus alumnos. El pediatra debe colaborar, asesorar, conjuntamente con el equipo psicopedagógico, y apoyar en todo el proceso.

\section{Iniciativas para cambiar la situación}

El acoso escolar debe ser evaluado y tratado de forma global, entendiendo que todos los participantes tienen algún sufrimiento y zonas erróneas.

Para poder "salvar la cara" los humanos "nos contamos historias" que nos permiten hacer cosas que de otra manera no podríamos justificar. Sin reconocer esto (que todos hacemos) no podrían explicarse las demonizaciones periódicas que hacemos unos de otros: judíos, musulmanes, inmigrantes, homosexuales o de los contrarios en el fútbol (por ejemplo). Este error cognitivo nos permite matar, linchar, agredir o excluir al "diferente" con el consentimiento tácito o 
explícito del grupo ideológico. Es así cómo se construyen los "chivos expiatorios". Además se facilita la ausencia de empatía con la víctima (cosificada o deshumanizada) permitiendo que sea ella quien cargue con las ansiedades y frustraciones del grupo. Por ello, hay que abordarlo de forma grupal (y social).

D. Olweus ${ }^{3}$, en 1980, señaló tres factores en el ámbito familiar que a su juicio considera decisivos para el desarrollo de modelos de agresión entre los alumnos: 1) actitud emotiva de los padres negativa, socializando a sus hijos sin afecto ni dedicación; 2) la ausencia de límites y permisividad ante la conducta agresiva de su hijo, y 3) uso del castigo físico y el maltrato emocional para afirmar su autoridad. También como se ha comentado anteriormente, los padres que sobreprotegen a su hijo y le dificultan desarrollar habilidades sociales pueden sin querer, favorecer su victimización. Como casi todo en educación, los extremos (muy permisivos-déspotas), nunca suelen ser adecuados.

En cuanto a los factores dependientes de la escuela, las condiciones de riesgo y protección que sobre ella influyen son múltiples y complejos ${ }^{11}$. Pero se sabe que en aquellas instituciones que tienen bien establecidas formas de prevenir, denunciar y actuar eficazmente ante este fenó- meno hay menos problemas y se abordan antes de que se cronifiquen. También se ha demostrado menor repertorio de actos violentos si se fomentan los vínculos seguros entre profesores y alumnos y entre los alumnos entre sí. Deben existir límites bien especificados y una disciplina coherente y basada en el afecto y el apoyo ${ }^{12}$. Que haya una filosofía de centro que tienda a la excelencia y cuente con un currículo de no violencia contribuye a disminuirlo. Por el contrario, las escuelas que tienen estructuras jerárquicas sin participación de la comunidad educativa, carecen de normas de conducta establecidas o tienen un sistema disciplinario inconsistente, laxo, ambiguo o extremadamente rígido pueden favorecer que surjan y se mantengan situaciones violentas en sus aulas. Son este tipo de instituciones las que suelen negar la importancia de lo que ocurre o no les parece que sea su responsabilidad.

Aunque excede el ámbito pediátrico entrar en el aula a trabajarlo, los profesores responsables deben abordarlo utilizando métodos pedagógicos adecuados. Para ello necesitan estar sensibilizados con el tema y saber qué hacer. Muchas veces lo ignoran y esto puede ser la causa de que lo nieguen. Hay en la bibliografía varias guías útiles ${ }^{10}$.

Desde nuestra perspectiva, el educar a 
los padres para que socialicen con afecto y limites, pasen tiempo suficiente con sus hijos y se interesen por su vida, nunca es tiempo perdido. El desarrollar habilidades de comunicación no violenta ${ }^{13}$, el aprender desde pequeños que el respeto es necesario para convivir (y más en las sociedades plurales y multicreenciales de ahora) y que las diferencia y la diversidad forman parte de la vida, debe formar parte de los mínimos de nuestra educación ${ }^{14}$.

Que los niños tengan buena autoestima, sean asertivos y aprendan a solucionar de forma prosocial el conflicto les dará herramientas para pararlo y cuestionarlo. Saber que algunas características físicas, sociales o psíquicas lo favorece (niños prematuros, impopulares, pesados, de otras culturas, hiperactivos, etc.) debería ayudar a ponernos en guardia para prevenir su aparición ${ }^{15}$. El tener bien desarrollada la empatía, ponerse en el lugar del otro y ser conscientes del sufrimiento (gratuito) que infligimos, nos hará mejores, menos egocéntricos y más felices. $Y$ ayudará a que la sociedad que formemos sea más amable.

Nuestra experiencia (sesgada) en los casos que vemos, es que se ha intervenido tarde (o no se ha hecho nada) y la víctima ha sufrido un daño difícil de recomponer $^{16}$. Al final la mayor parte de las veces acaba abandonando el centro. Hay información suficiente para conocer el efecto devastador que esta violencia produce. El pediatra puede ser una figura de detección y apoyo en algunos casos. Sin duda la "tolerancia cero" debe ser promovida desde todos los organismos implicados y tiene que estar presente de forma explícita en el currículo escolar y familiar: "Nosotros, no nos comportamos así"17.

\section{Nota de los autores}

En el texto, cada vez que nos referimos a personas, hacemos referencia indistintamente a ambos sexos.

\section{Bibliografía}

1. Cipolla CM. Las leyes fundamentales de la estupidez humana. Lampre Editorial [en línea] [consultado el 09/11/2011]. Disponible en http:// piensoparatodos.com/cosas/estupidez \% 20humana.pdf
2. Marina JA. La inteligencia fracasada: teoría y práctica de la estupidez humana. Barcelona: Anagrama; 2004.

3. Olweus D. Acoso escolar, "bullying", en las escuelas: hechos e intervenciones [en línea] [consultado el 09/11/2011]. Disponible en www.aco somoral.org/pdf/Olweus.pdf 
4. Defensor del Pueblo. Violencia escolar: el maltrato entre iguales en la Educación Secundaria Obligatoria 1999-200. Madrid: Defensor del Pueblo; 2007 [en línea] [consultado el 09/11/2011]. Disponible en www.defensordelpueblo.es/es/Do cumentacion/Publicaciones/monografico/Do cumentacion/Informe_violencia_escolar_ESO.pdf

5. Oñate Cantero A, Piñuel, Zabal I. Informe Cisneros X. Violencia y acoso escolar en España. Instituto de Innovación educativa y desarrollo directivo. 2007 [en línea]. Parcialmente disponible en www.fapacne.com/publicaciones/acoso-esco lar/acoso-escolar.pdf

6. Viera M, Fernández I, Quevedo G. Violence, bullying and counselling in the iberican peninsula. In: Roland E, Munthe E. Bullying: an international perspective. London: Fulton; 1989.

7. Ortega R. La convivencia escolar: qué es y cómo abordarla. Sevilla: Consejería de Educación y Ciencia. Junta de Andalucía; 1998.

8. Ortega R. Agresividad injustificada, bullying y violencia escolar. Madrid: Alianza Editorial; 2010.

9. Avilés JM. Bullying. Intimidación y maltrato entre el alumnado. 2002. Stee-Eilas [en línea] [consultado el 09/11/2011]. Disponible en www. educacionenvalores.org/IMG/pdf/bullying CAST.pdf

10. Collell J, Escudé C. El maltrato entre alumnos/as (Bullying) Guía para las familias [en línea] [consultado el 09/11/2011]. Disponible en www. xtec.es/ jcollell/

11. Díaz Aguado MJ. Por qué se produce la violencia escolar y cómo prevenirla. Rev Iberoam Educ. 2005;37:17-47.

12. Henderson N, Milstein M. Resiliencia en la Escuela. Barcelona: Paidós; 2006.

13. Rosenberg MB. Comunicación no violenta. Barcelona: Urano; 2000.

14. Marina JA. Aprender a convivir. Madrid: Ariel; 2006.

15. Mooij T. Por la seguridad en la escuela. Rev Educación. 1997;313:29-52.

16. Morán C. Intervención cognitivo-conductual en el acoso escolar: un caso de bullying. Anuario Psicología Clín Salud. 2006;2:51-6.

17. Garaigordobil $M$, Oñederra JA. La violencia entre iguales. Revisión teórica y estrategias de intervención. Madrid: Pirámide; 2010. 